\title{
On the fundamental group of compact homogeneous manifolds carrying an invariant fat distribution*
}

\author{
A. Lotta
}

\begin{abstract}
We show that a homogeneous space of a compact Lie group carrying an invariant fat distribution must have finite fundamental group.

MSC (2010): Primary: 53C30, 58A30; Secondary: 32V05.
\end{abstract}

Keywords: fat distribution, strongly bracket generating distribution, Levi-Tanaka form, homogeneous CR manifold.

\section{Introduction}

The aim of this note is to prove the following:

Theorem 1.1 Let $M=G / H$ be a homogeneous space of a compact Lie group $G$. If $M$ admits a $G$-invariant fat distribution, then $\pi_{1}(M)$ is finite.

In order to explain the "fat" assumption, we recall the relevant notions involved. We shall denote by $\Gamma E$ the module of smooth sections of a vector bundle $E$. Given a vector subbundle $D \subset T M$ of the tangent bundle of a smooth manifold $M$ (or equivalently a smooth distribution), the corresponding Levi-Tanaka form $\mathfrak{L}_{D}$ is the skew-symmetric $C^{\infty}(M)$-bilinear map

$$
\mathfrak{L}_{D}: \Gamma D \times \Gamma D \rightarrow \Gamma(T M / D)
$$

defined by

$$
\mathfrak{L}_{D}(X, Y):=\pi[X, Y]
$$

*Post-print version. Published source: Arch. Math. (Basel) 108 (2017), no. 6, 625628, DOI 10.1007/s00013-017-1021-8 
where $\pi: T M \rightarrow T M / D$ is the canonical projection. The classical theorem of Frobenius states that $D$ is an integrable distribution iff $\mathcal{L}_{D}=0$. We recall that $D$ is called Levi nondegenerate provided

$$
\left(\mathcal{L}_{D}\right)_{x}: D_{x} \times D_{x} \rightarrow T_{x} M / D_{x} \text { is nondegenerate for each } x \in M,
$$

that is

$$
\left\{v \in D_{x}:\left(\mathcal{L}_{D}\right)_{x}(v, w)=0 \text { for every } w \in D_{x}\right\}=\{0\} .
$$

Now, by definition, a fat distribution is one for which the following stronger condition holds:

$$
\left(\mathcal{L}_{D}\right)_{x}(v,-) \text { is onto for each } x \in M \text { and every nonzero } v \in D_{x} \text {. }
$$

We refer the reader to [5] for a recent analysis of the differential geometry of fat distributions, along the line of research started in [9]. We also remark that this notion has been intensively used in the context of sub-Riemannian geometry, where the term often used is "strongly bracket generating"; indeed, it is equivalent to requiring that, for any smooth nonvanishing section $X$ of $D, \Gamma T M$ is spanned by $X$ and $[X, D]$. See [8] and also [2].

If $D$ has codimension one, fatness reduces to Levi nondegeneracy. As regards this case, we mention the following application of our result in the context of $C R$ geometry:

Corollary 1.2 Let $(M, H M, J)$ be a connected, compact homogeneous $C R$ manifold of hypersuface type with nondegenerate Levi form. Then $\pi_{1}(M)$ is finite.

This has been already proved in [3] and, in the strongly pseudo convex case, in [4]. We also refer the reader to these papers for the basic notions of $C R$ geometry. Our proof appears to be more elementary. In order to apply Theorem 1.1 to the holomorphic distribution $D=H M$, it suffices to use the fact that, under the assumptions of the corollary, $M$ always admits a compact transitive group $G$ of $C R$ automorphisms: a direct proof of this statement has been provided in [7].

\section{Proof of Theorem 1.1}

Fix a bi-invariant metric $g$ on $G$; denote by $\langle\cdot, \cdot\rangle$ the corresponding $A d(G)$ invariant inner product on the Lie algebra $\mathfrak{g}$. We consider the induced 
$G$-invariant normal metric on $G / H$, denoted by the same symbol, turning $(G / H, g)$ into a naturally reductive homogeneous Riemannian manifold with nonnegative sectional curvature (cf. e.g. [6, Corollary X.3.6]). We claim that the existence of a $G$-invariant fat distribution forces the Ricci tensor of $g$ to be positive definite, so that (by homogeneity) Myers' Theorem applies yielding that $\pi_{1}(M)$ must be finite. To prove our claim, let $o=\pi(e)$, $\pi: G \rightarrow G / H$ being the canonical projection; the reductive decomposition of the Lie algebra $\mathfrak{g}$ of $G$ is

$$
\mathfrak{g}=\mathfrak{h} \oplus \mathfrak{m}
$$

where $\mathfrak{h}=\operatorname{Lie}(H)$ and $\mathfrak{m}=\mathfrak{h}^{\perp}$ is the orthogonal complement of $\mathfrak{h}$ with with respect to $\langle\cdot, \cdot\rangle$; the Ricci tensor of $g$ at $o$ is given, modulo the standard identification $T_{o} M \cong \mathfrak{m}$, by

$$
\operatorname{Ric}_{o}(X, X)=\frac{1}{4} \sum_{i=1}^{n}\left\|\left[X, E_{i}\right]_{\mathfrak{m}}\right\|^{2}+\sum_{i=1}^{n}\left\|\left[X, E_{i}\right]_{\mathfrak{h}}\right\|^{2}
$$

where $n=\operatorname{dim}(M)$ and $\left\{E_{i}\right\}$ is an orthonormal basis of $\mathfrak{m}$. This formula is easily checked via a straightforward computation using Proposition 3.4 in [6, Ch. X, p. 202].

Now, consider the canonical $G$-invariant connection $\nabla$ on the homogeneous space $G / H$, relative to the reductive decomposition (1) (cf. [6, Ch. $\mathrm{X}$, p. 192]). We recall that in this setting $\nabla$ is a metric connection with totally skew-symmetric torsion, i.e. its torsion tensor $T$, considered as a $(0,3)$ tensor field, is a 3 -form (see also [10] or [1]). Moreover, $T$ is determined at $o$ by

$$
T_{o}(X, Y)=-[X, Y]_{\mathfrak{m}}, \quad X, Y \in \mathfrak{m}
$$

We shall denote by $\mathfrak{z}$ the null space of the skew-symmetric bilinear map $T_{o}: \mathfrak{m} \times \mathfrak{m} \rightarrow \mathfrak{m}$. Now, we consider a $G$-invariant distribution $D$ on $M$. According to Proposition X.2.7 in [6], because of the $G$-invariance, $D$ must be parallel with respect to $\nabla$. Hence, after the canonical identification

$$
T M / D \cong D^{\perp}
$$

allowed by the metric $g$, the Levi form $\mathfrak{L}: \Gamma D \times \Gamma D \rightarrow \Gamma D^{\perp}$ of $D$ can be expressed by means of the torsion $T$ as follows:

$$
\mathfrak{L}(X, Y)=-P T(X, Y) \quad X, Y \in \Gamma D
$$

where $P: T M \rightarrow D^{\perp}$ is the orthogonal projection with respect to $g$. 
Assume henceforth that $D$ is fat. Hence, denoting by $\mathfrak{s}$ the subspace $D_{o}$ of $\mathfrak{m}$, the skew-symmetric bilinear map

$$
\mathfrak{L}_{o}: \mathfrak{s} \times \mathfrak{s} \rightarrow \mathfrak{s}^{\perp}
$$

satisfies that $\mathfrak{L}_{o}(X,-)$ is onto for each non null vector $X \in \mathfrak{s}$. We claim that this forces $\mathfrak{z}=\{0\}$.

According to (3), and using the fact that $T$ is a 3 -form, we get, for every $X, Y \in \mathfrak{s}$ and $\xi \in \mathfrak{s}^{\perp}$ :

$$
\left\langle\mathfrak{L}_{o}(X, Y), \xi\right\rangle=-\left\langle T_{o}(X, Y), \xi\right\rangle=-T_{o}(X, Y, \xi)=\left\langle T_{o}(X, \xi), Y\right\rangle .
$$

First of all we observe that this implies

$$
\mathfrak{z} \cap \mathfrak{s}=\{0\}=\mathfrak{z} \cap \mathfrak{s}^{\perp} .
$$

Indeed, the first equality holds since $\mathfrak{L}_{o}$ is nondegenerate, and the second one because $\mathfrak{L}_{o}$ is onto. Now, fix $U \in \mathfrak{z}$ and write

$$
U=X+\xi
$$

with $X \in \mathfrak{s}$ and $\xi \in \mathfrak{s}^{\perp}$. Then we must have $T_{o}(X, \xi)=0$, which by (4) yields

$$
\left\langle\mathfrak{L}_{o}(X, Y), \xi\right\rangle=0
$$

for every $Y \in \mathfrak{s}$. This implies that either $X=0$ or $\xi=0$, because $D$ is fat. In both cases, we conclude that $U=0$. Hence, by (2), the Ricci tensor is positive definite as claimed.

\section{References}

[1] I. AgRicola: The Srní lectures on non-integrable geometries with torsion, Arch. Math. (Brno) 42 (2006), suppl., 5-84.

[2] D. M. Almeida: On fat sub-Riemannian symmetric spaces in codimension three, Differential Geom. Appl. 24 (2006), no. 2, 178-190.

[3] H. Azad; A. Huckleberry; W. Richthofer: Homogeneous CRmanifolds, J. Reine Angew. Math. 358 (1985), 125-154.

[4] D. Burns; S. Shnider: Spherical hypersurfaces in complex manifolds, Invent. Math. 33 (1976), no. 3, 223-246. 
[5] A. Kaplan; M. Subils: On the equivalence problems for bracketgenerating distributions. Hodge theory, complex geometry, and representation theory, 157-171, Contemp. Math. 608, Amer. Math. Soc., Providence, RI, 2014.

[6] S. Kobayashi; K. Nomizu: Foundations of differential geometry. Vol II. Interscience Publishers John Wiley \& Sons, Inc., New York-LondonSydney 1969.

[7] A. SpIRo: Groups acting transitively on compact CR manifolds of hypersurface type, Proc. Amer. Math. Soc. 128 (2000), no. 4, 11411145 .

[8] R. S. Strichartz: Sub-Riemannian geometry, J. Differential Geom. 24 (1986), no. 2, 221-263.

[9] N. TANAKA: On the equivalence problems associated with simple graded Lie algebras, Hokkaido Math. J. 8 (1979), no. 1, 23-84.

[10] F. TRICERRI; L. VANHECKE: Naturally reductive homogeneous spaces and generalized Heisenberg groups, Compositio Math. 52 (1984), no. 3, 389-408.

Author's address:

Antonio Lotta

Dipartimento di Matematica

Università degli Studi di Bari Aldo Moro

Via E. Orabona 4, 70125 Bari

ITALY

e-mail: antonio.lotta@uniba.it 